Mon. Not. R. Astron. Soc. 000, 000-000 (0000) Printed 26 November $2018 \quad$ (MN LATEX style file v2.2)

\title{
Making Galaxies in a Cosmological Context: The Need for Early Stellar Feedback
}

\author{
G. S. Stinson ${ }^{1 \star}$, C. Brook ${ }^{2}$, A. V. Macciò ${ }^{1}$, J. Wadsley ${ }^{3}$, T. R. Quinn ${ }^{4}$, \\ H. M. P. Couchman ${ }^{3}$ \\ ${ }^{1}$ Max-Planck-Institut für Astronomie, Königstuhl 17, 69117, Heidelberg, Germany \\ ${ }^{2}$ Departamento de Físíca Teórica, Universidad Autónoma de Madrid, E-28049 Cantoblanco, Madrid, Spain \\ ${ }^{3}$ Department of Physics and Astronomy, McMaster University, Hamilton, Ontario, L8S 4M1, Canada \\ ${ }^{4}$ Astronomy Department, University of Washington, Box 351580, Seattle, WA, 98195-1580
}

26 November 2018

\begin{abstract}
We introduce the Making Galaxies in a Cosmological Context (MaGICC) program of smoothed particle hydrodynamics ( $\mathrm{SPH})$ simulations. We describe a parameter study of galaxy formation simulations of an $\mathrm{L}^{\star}$ galaxy that uses early stellar feedback combined with supernova feedback to match the stellar mass-halo mass relationship. While supernova feedback alone can reduce star formation enough to match the stellar mass-halo mass relationship, the galaxy forms too many stars before $z=2$ to match the evolution seen using abundance matching. Our early stellar feedback is purely thermal and thus operates like a UV ionization source as well as providing some additional pressure from the radiation of massive, young stars. The early feedback heats gas to $>10^{6} \mathrm{~K}$ before cooling to $10^{4} \mathrm{~K}$. The pressure from this hot gas creates a more extended disk and prevents more star formation prior to $z=1$ than supernovae feedback alone. The resulting disk galaxy has a flat rotation curve, an exponential surface brightness profile, and matches a wide range of disk scaling relationships. The disk forms from the inside-out with an increasing exponential scale length as the galaxy evolves. Overall, early stellar feedback helps to simulate galaxies that match observational results at low and high redshifts.
\end{abstract}

Key words: galaxies: formation - galaxies:ISM - hydrodynamics - methods: N-body simulation

\section{INTRODUCTION}

As matter collapses in the early Universe, gas initially heats as kinetic energy is converted into thermal (Rees \& Ostriker 1977; Birnboim \& Dekel 2003). In massive halos, the gas reaches temperatures where its cooling time becomes longer than the Hubble time (Rees \& Ostriker 1977), which results in a theoretical maximum galaxy mass. However, in the centers of galaxies, enough gas accumulates to increase the density to the point that the gas efficiently radiates and cools. Such cooling is unstable since the radiation leaves through the optically thin surrounding hot gas. This process leads to overcooling since as the hot gas cools, it stops providing pressure support for the surrounding gas, more dense gas is able to accumulate and cool even more efficiently White \& Rees 1978). This problem is known as the "overcooling catastrophe" (e.g. Balogh et al. 2001).

* Email: stinson 'at' mpia.de
The "overcooling catastrophe" has long plagued simulations of disk galaxy formation. Fully cosmological numerical galaxy simulations consistently contain a massive central concentration of stars (Navarro \& Benz 1991; Governato et al. 2004; Stinson et al. 2010; Scannapieco et al. 2012). This is evident in the central peak in rotation curves in simulated galaxies as well as in their surface brightness profiles.

Further evidence for simulations forming too many stars comes from recent studies matching observations of galaxy stellar masses with their dark matter halo masses. One method is the abundance matching technique. To lay the groundwork for this technique, Conroy et al. (2006) rank ordered halos by total halo mass from collisionless simulations and then did a rank ordering of galaxies based on their stellar mass from the Sloan Digital Sky Survey. The halos and galaxies were then divided in several mass bins. Conroy et al. (2006) found that the correlation functions between halos in certain mass bins were well matched by 
Stinson et al.

the correlation functions of galaxies in corresponding stellar mass bins. Several groups used this abundance matching technique to compare total halo masses with galaxy stellar masses (Conroy \& Wechsler 2009; Moster et al. 2010; Guo et al. 2010; Behroozi et al. 2010). Direct comparisons of stellar mass and total halo mass have also been done based on satellite dynamics Klypin \& Prada 2009; More et al. 2009, 2011) and weak lensing (Mandelbaum et al. 2009; Schulz et al. 2010). For a comparison of all these techniques, please see Figure 11 of Behroozi et al. (2010). Each method shows a good level of correspondence. Guo et al. (2010) and Sawala et al. (2011) showed that nearly all the simulations of galaxy formation have formed many more stars than abundance matching predicts.

Even without overcooling, van den Bosch et al. (2002) showed that the amount of low angular momentum material in collapsed collisionless halos exceeds the amount of low angular momentum material observed in disk galaxies. Consequently, this low angular momentum material needs to be removed from the center of the system.

Stellar feedback is the favored way of reducing star formation and launching outflows (Scannapieco et al. 2008; Schave et al. 2010). Dekel \& Silk (1986) showed that supernova feedback can eject gas from galaxies with virial velocities up to $100 \mathrm{~km} \mathrm{~s}^{-1}$. Semi-analytic models have found that significant amounts of stellar feedback are required to match the low mass end of the luminosity function (Somerville \& Primack 1999; Benson et al. 2003; Bower et al. 2006, 2008, 2012). Dutton \& van den Bosch (2009) showed that stellar feedback can remove low angular momentum material.

In hydrodynamical simulations, two methods are commonly used to model stellar feedback. One is kinetic feedback that adds velocity kicks to gas particles to remove them from the inner regions of galaxy disks (Springel \& Hernquist 2003; Oppenheimer \& Davé 2006; Dalla Vecchia \& Schave 2008). The other is thermal feedback in which stars simply heat gas particles and allow the adiabatic work of the particles to push other gas out of the way Gerritsen \& Icke 1997; Thacker \& Couchman 2000; Kawata \& Gibson 2003; Stinson et al. 2006).

Since stars form in dense regions, the cooling times of the surrounding gas are short, and without help, the gas will quickly radiate away all the supernova energy (Katz 1992). In real galaxies, the amount of gas necessary to exert a dynamical influence on the ISM small. In simulations, such small amounts of gas are difficult to model, so a common technique has been to turn off cooling for a limited amount of time (Gerritsen \& Icke 1997; Thacker \& Couchman 2000; Brook et al. 2004; Stinson et al. 2006).

It is not clear that kinetic and thermal feedback have significantly different effects than one another. Durier \& Dalla Vecchia (2012) showed that kinetic feedback has the same effect as thermal feedback when the hydrodynamics is left turned on. However, in many implementations of kinetic feedback, the hydrodynamic processes are disabled for a set period of time to maintain numerical convergence (Springel \& Hernquist 2003; Oppenheimer \& Davé 2006).

It is also possible that thermal feedback does not need to rely on disabling cooling. Dalla Vecchia \& Schave (2012) recently showed that thermal feedback can have a significant dynamical effect if the total energy of all the supernovae ex- plosions is injected into one particle at one time. This large, one time energy deposition raises the particle's temperature high enough that its cooling time lengthens enough for the feedback to have a dynamical effect.

For the first time in simulations, Governato et al. (2010) showed that thermal stellar feedback can remove low angular momentum material from dwarf galaxies, creating galaxies with slowly rising rotation curves. Brook et al. (2011) showed that the ejected low angular momentum material becomes part of a galactic fountain and can be reaccreted onto the disk with higher angular momentum than it left. Sawala et al. (2011) showed that even using a high density threshold for star formation that made the stellar feedback more efficient, these simulations produced more stars than is predicted by the stellar mass-halo mass relationship.

Three other recent simulations have shown success at forming realistic $L^{\star}$ disk galaxies. Guedes et al. (2011) used high resolution and a high amount of supernova feedback to produce a realistic disk galaxy. Agertz et al. (2011) suggested that using a low star forming efficiency is helpful for creating extended disks. They also use models that increase the amount of supernova energy deposited above the canonical $10^{51}$ erg per supernova. These simulations do the best job flattening out the central region of their rotation curve. McCarthy et al. (2012) also form galaxies with flat rotation curves in a large, low resolution cosmological volume. They employ a kinetic feedback scheme that uses less than the canonical $10^{51}$ erg supernova energy, though see Kav et al. (2002) for a demonstration of how kinetic feedback provides a larger effect than thermal feedback. The galaxies each contain a few thousand particles and employ a gravitational softening of $\sim 1 \mathrm{kpc}$, which makes an examination of the detailed structure of the galaxies challenging.

These simulations show that it is possible that more feedback than the canonical $10^{51} \mathrm{erg}$ is required. Instead of simply increasing the amount of energy released by supernovae feedback, we note that Murray et al. (2010) showed that there can be significant feedback effects from stars before they explode as supernovae. Hopkins et al. (2011) incorporated a kinetic radiation pressure feedback into simulations of isolated disk galaxies and found that the feedback could strongly regulate star formation. While we do not have the resolution in cosmological simulations to implement a similar kinetic scheme, we implement a scheme based on thermal pressure to provide feedback during the time between when stars are formed and the first SN star exploding.

Using this feedback prescription, Macciò et al. (2012) showed that the feedback removes low angular momentum dark matter in galaxies up to nearly $\mathrm{L}^{\star}$, producing cored dark matter density profiles. Stinson et al. (2012) showed that the metal rich outflows created by this feedback match observations of OVI in the circum-galactic medium of star forming galaxies. Brook et al. (2012b) also showed that a sample of lower mass galaxies form disks that follow a wide range of disk scaling relationships.

Here, we present a detailed study of how varying the key free parameters in our simulations affect the morphology and evolution of galaxies. In 2 , we describe the galaxy we model and the physics used in the simulation. In $\$ 3$ we show how varying parameters affects the mass of stars formed, the morphology of the galaxy, and how the galaxy evolved. 


\section{SIMULATIONS}

We use g1536, a cosmological zoom simulations drawn from the McMaster Unbiased Galaxy Simulations (MUGS), in our parameter study. See Stinson et al. (2010) for a complete description of the creation of the initial conditions. g1536 has a total mass of $7 \times 10^{11} \mathrm{M}_{\odot}$, a spin parameter of 0.017 , and a last major merger at $z=2.9$. Using the physics employed in the original MUGS simulations, g1536 also formed an exponential disk with an classical bulge with a central surface brightness of $\mu_{i}=14$, a total face-on magnitude of $\mathrm{M}_{r}=-21.7$ and $g-r$ colour of 0.62 .

Using the smoothed particle hydrodynamics (SPH) code GASOLINE (Wadsley et al. 2004), we varied the stellar feedback to reduce star formation to the amount prescribed in Moster et al. (2012). We first increased the energy input from supernovae to $10^{51}$ erg and changed the initial stellar mass function (IMF) from what was described in Kroupa et al. (1993) to an IMF that contains more massive stars as described in Chabrier (2003). Neither of these changes sufficiently limited star formation, so we turned to a source of energy that is often ignored in cosmological simulations, the luminosity emitted by massive stars before they explode as supernovae. Hopkins et al. (2011) has shown that the inclusion of radiation pressure in high resolution simulations can reduce star formation. The supernovae explosions in our previous simulations did not happen until 4 Myr after the formation of a stellar population. However, molecular clouds are disrupted much sooner than this, so the inclusion of this early stellar feedback is necessary to reduce star formation before supernovae start exploding.

\section{$2.1 \quad$ Gas Cooling}

In addition to energy input, the gas cooling has a large effect on how many stars form. The cooling used in this paper is described in detail in Shen et al. (2010). It was calculated using CLOUDY (version 07.02; Ferland et al. (1998)) including photoionization and heating from the Haardt \& Madau (2005) UV background, Compton cooling, and hydrogen, helium and metal cooling from 10 to $10^{9} \mathrm{~K}$. Shen et al. (2010) showed that $T>10^{4} \mathrm{~K}$ metal cooling including the photoionization effects of the UV background reduces cooling by an order of magnitude from purely collisional cooling rates Sutherland \& Dopita (1993).

In the dense, interstellar medium gas, we do not impose any shielding from the extragalactic UV field. While the cosmic UV background used is lower than the typical UV field within galaxies, it does provide heating similar to that expected in the ISM.

When metal cooling is included below $10^{4} \mathrm{~K}$, it leads to the well-known two-phase instability of the ISM Field 1965; Field et al. 1969), which produces densities over $100 \mathrm{~cm}^{-3}$ and temperatures of $100 \mathrm{~K}$ in these simulations. These values correspond to a Jeans mass of $<6000 \mathrm{M}_{\odot}$ and a Jeans length of $<8$ pc. Each of these values are far below both the gas particle mass, $2 \times 10^{5} \mathrm{M}_{\odot}$, or $310 \mathrm{pc}$ softening length. In reality, this phase would become even denser, self-shielded, and ultimately molecular, but we cannot resolve this process and must therefore estimate the star formation rate from the resolved dense gas. To avoid issues with Jeans fragmentation, a minimum pressure is established in the gas as described in
Robertson \& Kravtsov (2008). A maximum density is also set to $400 \mathrm{~cm}^{-3}$ using a minimum smoothing length $\frac{1}{4}$ of the 310 pc gravitational softening.

\subsection{Star Formation and Feedback}

The simulations use a common recipe for star formation described in Stinson et al. (2006) that we summarize here. Stars form from cool $(T<15,000 \mathrm{~K})$, dense gas. The metal cooling readily produces dense gas, so the star formation density threshold is set to the maximum density at which gravitational instabilities can be resolved, $\frac{32 M_{\text {gas }}}{\epsilon^{3}}\left(n_{t h}>9.3\right.$ $\left.\mathrm{cm}^{-3}\right)$, where $M_{\text {gas }}=2.2 \times 10^{5} \mathrm{M}_{\odot}$ and $\epsilon$ is the gravitational softening $(310 \mathrm{pc})$. Such gas is converted to stars according to the equation

$\frac{\Delta M_{\star}}{\Delta t}=c_{\star} \frac{M_{g a s}}{t_{d y n}}$

Here, $\Delta M_{\star}$ is the mass of the star particle formed, $\Delta t$ is the timestep between star formation events, $8 \times 10^{5} \mathrm{yr}$ in these simulations, and $t_{d y n}$ is the gas particle's dynamical time. $c_{\star}$ is the efficiency of star formation, in other words, the fraction of gas that will be converted into stars during $t_{d y n}$.

Stars feed both energy and metals back into the interstellar medium gas surrounding the region where they formed. Supernova feedback is implemented using the blastwave formalism described in Stinson et al. (2006). In this, Type II supernovae are assumed to explode due to the core collapse at the end of lifetime of stars greater than $8 \mathrm{M}_{\odot}$. Stellar lifetimes are based on the Padua lifetimes (Raiteri et al. 1996). Since the gas receiving the energy is dense, it would quickly be radiated away due to its efficient cooling. For this reason, cooling is disabled for particles inside the blast region

$r_{C S O}=10^{1.74} E_{51}^{0.32} n_{0}^{-0.16} \tilde{P}_{04}^{-0.20} \mathrm{pc}$

and for the length of time

$t_{C S O}=10^{6.85} E_{51}^{0.32} n_{0}^{0.34} \tilde{P}_{04}^{-0.70} \mathrm{yr}$

given in McKee \& Ostriker (1977).

\subsection{Early Stellar Feedback}

In an effort to provide a more physical model for stellar feedback, radiation energy from massive stars is now considered. Heating is introduced immediately after stars form based on how much star light is radiated. The early energy input from high mass stars has been entirely ignored in most previous cosmological simulations. In such cases, 4 Myr pass after stars form before the first supernova explodes (some simulations use even longer delays, see Slyz et al. 2005; Dalla Vecchia \& Schaye 2012), during which time stars can continue forming without any affect of stellar feedback.

Hopkins et al. (2011) describes how such energy could be considered using a kinetic scheme where the radiation pressure drives winds out of massive star clusters. Our simulations do not have the resolution to use kinetic feedback because it requires the choice of a direction. A kinetic implementation of feedback requires many particles to represent a molecular cloud so that it can be torn apart. In our 
Table 1. Simulation data

\begin{tabular}{llllllll}
\hline Name & color & $\mathrm{c}^{a}$ & $\epsilon_{e s f}{ }^{b}$ & $C_{t d}{ }^{c}$ & $\mathrm{IMF}^{d}$ & $\mathrm{M}_{\star}\left[\mathrm{M}_{\odot}\right]^{e}$ & $M_{V}^{f}$ \\
\hline Fiducial & red & 0.1 & 0.1 & 0.05 & $\mathrm{C}$ & $2.3 \times 10^{10}$ & -20.6 \\
MUGS & black & 0.05 & 0 & $0.05^{g}$ & $\mathrm{~K}$ & $8.3 \times 10^{10}$ & -21.4 \\
Low Diffusion & green & 0.1 & 0.1 & $0.01^{g}$ & $\mathrm{C}$ & $3.0 \times 10^{10}$ & -21.1 \\
High Diffusion & yellow & 0.1 & 0.175 & $0.05^{g}$ & $\mathrm{C}$ & $2.5 \times 10^{10}$ & -21.4 \\
No ESF & magenta & 0.1 & 0 & 0.05 & $\mathrm{C}$ & $3.4 \times 10^{10}$ & -19.5 \\
120\% SN energy & cyan & 0.1 & 0 & 0.05 & $\mathrm{C}$ & $1.8 \times 10^{10}$ & -20.0 \\
High ESF & blue & 0.1 & 0.125 & 0.05 & $\mathrm{C}$ & $1.1 \times 10^{10}$ & -19.9 \\
\hline
\end{tabular}

$a$ Star forming efficiency

$b$ Early Stellar Feedback Efficiency

$c$ Thermal Diffusion coefficient

$d$ Initial Mass Function: C=Chabrier (2003); K= Kroupa et al. (1993)

$e \mathrm{M}_{\star}$ is the total stellar mass

$f$ V-band magnitude

$g$ All particles diffused thermal energy including those with cooling shut off

simulations, only one or a few particles represent a molecular cloud, so we utilize thermal pressure, which is isotropic. Using thermal feedback provides pressure support and increases gas temperatures above the star formation threshold to decrease star formation. We do not disable cooling during these early times since massive young stars radiate a large amount of energy. Thus, after heating the gas to $T>10^{6} \mathrm{~K}$, the gas rapidly cools to $10^{4} \mathrm{~K}$, which creates a lower density medium than if the gas were allowed to continue cooling until supernovae exploded.

To model the luminosity of stars, a simple fit of the mass-luminosity relationship observed in binary star systems by Torres et al. (2010) is used:

$\frac{L}{L_{\odot}}= \begin{cases}\left(\frac{M}{M_{\odot}}\right)^{4} & , M<10 M_{\odot} \\ 100\left(\frac{M}{M_{\odot}}\right)^{2} & , M>10 M_{\odot}\end{cases}$

Typically, this relationship leads to $2 \times 10^{50}$ erg of energy being released from the high mass stars per $\mathrm{M}_{\odot}$ of the entire stellar population over the $4 \mathrm{Myr}$ between the star's formation and the commencing of SNII (SNII inject $\sim 10^{49} \mathrm{erg}$ per $\left.M_{\odot}\right)$.

Leitherer et al. (1999) found that approximately $10 \%$ of the total stellar flux is emitted in the UV, so we use with an early stellar feedback efficiency, $\epsilon_{e s f}=10 \%$. (Freyer et al. 2006) found that stellar photons do not couple efficiently with the surrounding ISM. Correspondingly, radiative cooling is not turned off for this form of energy input. The dense gas thus cools rapidly to $10^{4} \mathrm{~K}$ in the ISM, which mimics the formation an HII region. Though the dynamical effect is minimal, early stellar feedback effectively halts star formation in the region immediately surrounding a recently formed star. Using $\epsilon_{e s f}=10 \%$ limits star formation to the amount prescribed by the stellar mass-halo mass relationship.

\subsection{Diffusion}

Agertz et al. (2007) highlighted how long cold gas clumps can survive in a surrounding bath of hot gas in SPH relative to Eulerian grid simulations. In an effort to decrease this mixing time, Wadsley et al. (2008) implemented diffusion into SPH based on mixing that should result in shearing flows. The amount of mixing depends on the magnitude of the local velocity shear field multiplied by the square of a length comparable to the grid or resolution scale. Wadslev et al. (2008) showed that diffusion of thermal energy effectively limits the lifetime of the blobs that Agertz et al. (2007) described. In MUGS (Stinson et al. 2010), both metals and heat diffused using this scheme with a diffusion coefficient of 0.05 . The most striking effect of the thermal diffusion was that cold accretion filaments were heated and expanded. Cold blobs persisted in these simulations. Results of varying the thermal diffusion coefficient are presented in 3

During this study, it was determined that the heat diffusion severely reduced the efficiency of the adiabatic feedback scheme to drive outflows. Hot particles with their cooling shut off frequently passed cooler particles with their cooling turned on. Heat would diffuse from the hot to cold particles, reducing the temperature of the particles and thus the efficiency of the feedback. This would happen mostly as hot particles orbited around the disk and occasionally after particles had been ejected from the disk. Since the feedback is dependent on particles not cooling, we disabled thermal diffusion in interactions between particles in which either particle had its cooling shut off. We note that disabled cooling has the biggest effect in the dense disk. We find less than $1 \%$ of hot $\left(\mathrm{T}>10^{4} \mathrm{~K}\right)$ particles with their cooling disabled are outside the disk at $z=0$ and such particles are never found beyond $25 \mathrm{kpc}$ at any time.

Figure 1 shows how the star formation histories of the different implementations of thermal diffusion vary. In the fiducial model, the star formation declines until the end of the simulation. The scheme where all gas particles always diffused required a $75 \%$ increase in $\epsilon_{\mathrm{ESF}}$ to limit the star formation to the stellar mass-halo mass relationship. The shape of the star formation history for the high diffusion model is different. It increases throughout the history of the galaxy and begins to start a starburst over the last 1 Gyr. This shows how with too much thermal diffusion star formation acts as a positive feedback: the more stars form, the 


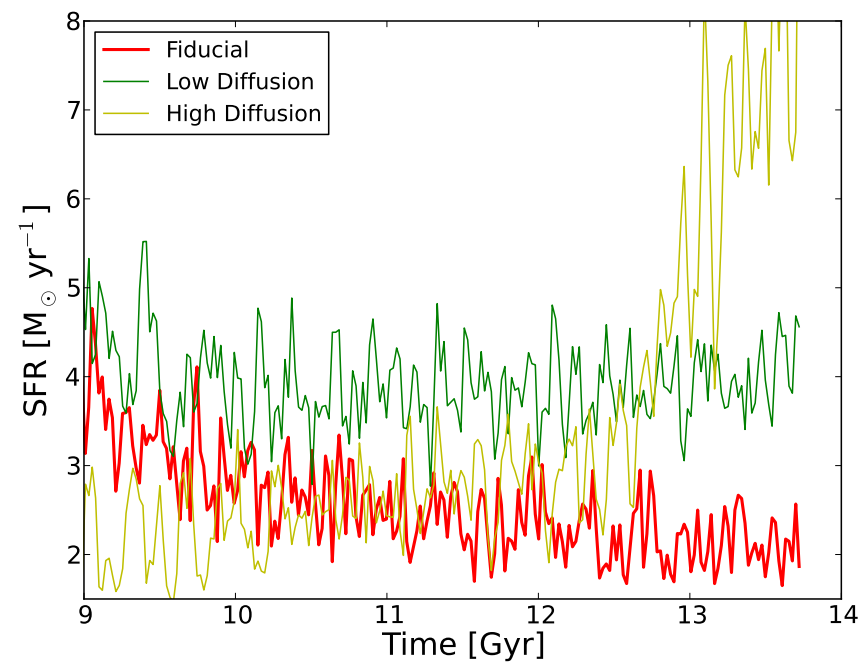

Figure 1. The star formation history of galaxies simulated with varying amounts of thermal diffusion. Here, the simulation evolves from left to right. This plot focuses on the last 5 Gyr of the simulations since that is when the biggest difference in star formation rate is seen.

more gas cools enabling more star formation. The low thermal diffusion simulation (green) demonstrates a milder increase in star formation rate at the end of the simulation, but it remains above the fiducial value. An examination of the simulation reveals that this is due to cold gas clouds cooling out of the hot halo as described in Maller \& Bullock (2004) and Kaufmann et al. (2009). In the revised treatment, the limited diffusion helps to limit the formation of cold blobs and reduces the star formation rate at late times in the simulation.

\section{RESULTS}

To find an optimal star formation and feedback recipe, we attempt to match the stellar mass-halo mass relationship. The parameters for all of our simulations are presented in Table 1. Figure 2 shows a mock observation of the fiducial galaxy. The image is $50 \mathrm{kpc}$ on a side and was created using the Monte Carlo radiative transfer code SunRISE Jonsson 2006). The image brightness and contrast are scaled using asinh as described in Lupton et al. (2004) since disks have an exponential surface brightness profiles meaning the images span a wide range in surface brightness. The image shows a thin, young stellar disk with clumpy and extended star formation, a dust lane, and a red bulge component $a$

\subsection{Total Mass}

Figure 3 shows the mass of the stellar component at $z=0$ as a function of halo mass. For the sake of clarity we have limited the number of simulations displayed to those which demonstrate our walk through parameter space. The coloured stars show the $z=0$ stellar and halo masses of

a Movies of the simulations can be found at
http://www.mpia.de/ $\sim$ stinson/magicc
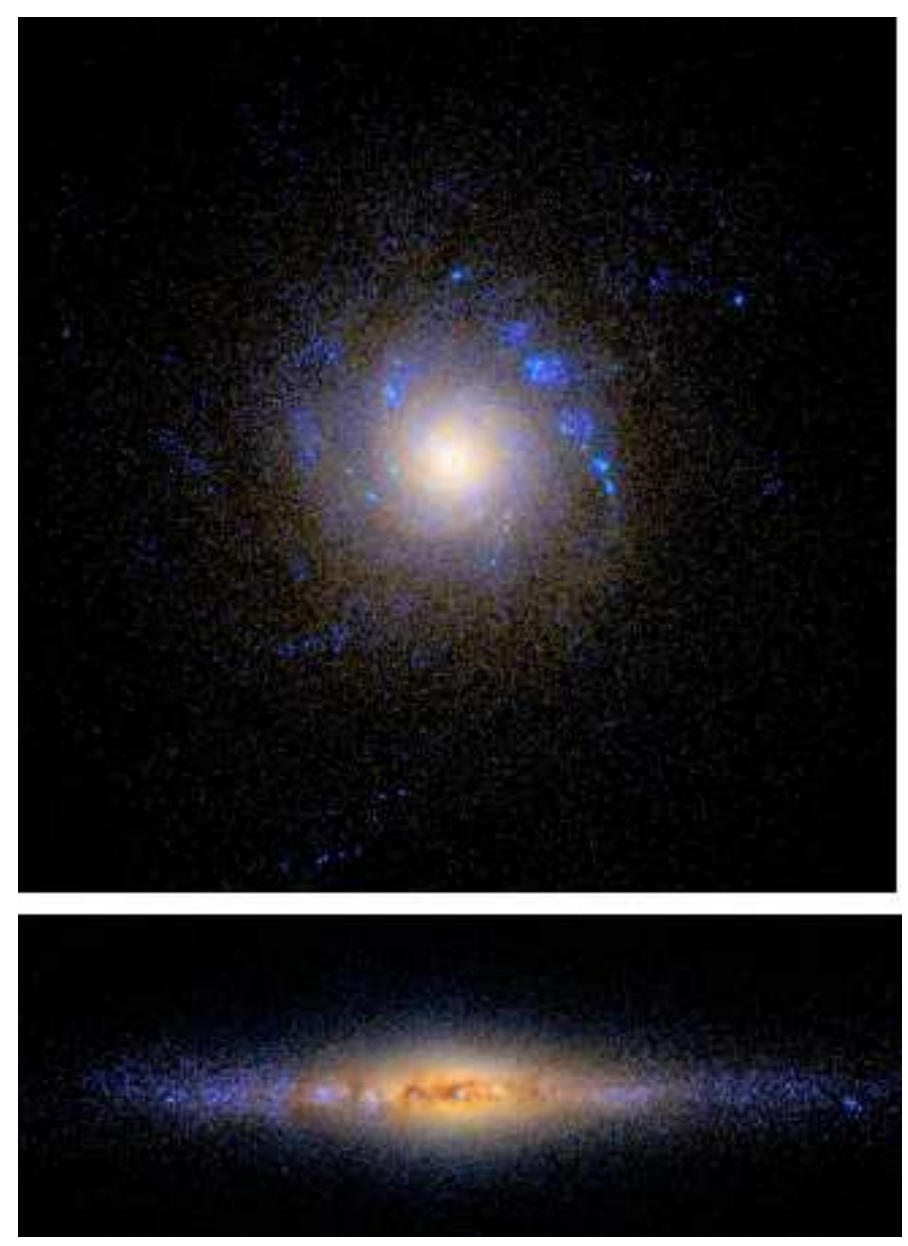

Figure 2. Face-on and edge-on images of the fiducial galaxy at $z=0$. The images are $50 \mathrm{kpc}$ on a side and were created using the Monte Carlo radiative transfer code SUNRISE. The image brightness and contrast are scaled using asinh as described in Lupton et al. (2004).

the simulations of g1536 used in this parameter study. Each $z=0$ point is accompanied by a line that shows the path along which it evolved through this diagram. A couple of lines are provided for reference. The dotted line is the cosmic baryon fraction of the total halo mass. The dashed line is the stellar mass halo mass relationship found in Moster et al. (2012). The black plus signs are the old results from MUGS. These lower feedback runs are similar to many other low feedback simulations where galaxies turn about half of a galaxy's baryons, as predicted by the cosmic baryon fraction, into stars.

The red star represents the best fit to the stellar masshalo mass relationship. It includes early stellar feedback with thermal diffusion disabled for particles with their cooling disabled. It uses a star formation efficiency, $c^{\star}=0.1$, an early stellar feedback efficiency, $\epsilon_{e s f}=0.1$, and a thermal diffusion coefficient, $C_{t d}=0.05$. The fiducial simulation evolves along a path generally below the $z=0$ Moster et al. (2012) relationship, which is shown in 3.3 to be similar to how the observed stellar to halo mass ratio evolves. A small change in the amount of feedback causes a significant change in the number of stars that form. When $\epsilon_{\text {esf }}$ increases from 0.1 to 


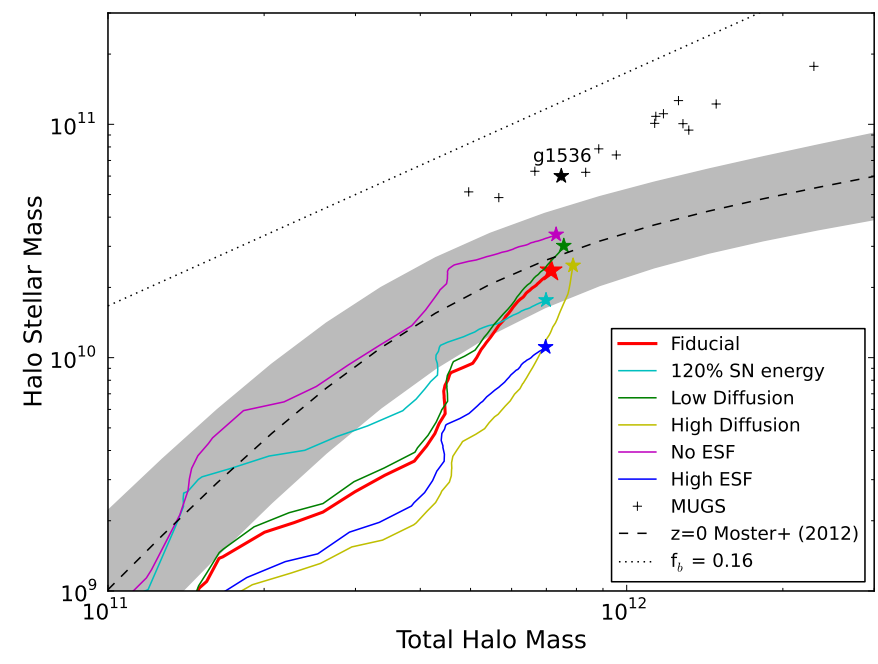

Figure 3. Stellar mass plotted as a function of halo mass. The abundance matching fit from Moster et al. (2012) and the cosmic baryon fraction lines are shown as reference. All the MUGS simulations formed too many stars along a line where half the baryons have turned into stars and half are in the hot gas halo. The simulations of g1536 are shown as coloured stars and lines. The legend provides a brief description of which simulation each of these colours represents, while a more extensive description can be found in Table 1. The fiducial simulation is coloured red and lies closest to the Moster et al. (2012) relationship. The yellow star representing the simulation where all gas particles diffuse thermal energy also lies close to the relationship line, but it is rapidly forming stars and will quickly overshoot the relationship. The blue point uses the same physics as the fiducial run, but increases the $\epsilon_{e s f}$ by $25 \%$ and forms far fewer stars. The magenta line shows a simulation run without early stellar feedback. While it ends up close to the fiducial run at $z=0$, it follows a dramatically different evolutionary path.

0.125 (represented in blue), the stellar mass decreases by a factor of 2 .

The magenta star and line represent the simulation in which only the supernova feedback was increased from the MUGS value of $4 \times 10^{50} \mathrm{erg}$ per supernova to $10^{51} \mathrm{erg}$. In this simulation, the thermal diffusion is turned off for particles with their cooling disabled. This change significantly decreases the mass of stars formed, though the stellar mass still lies above the Moster et al. (2012) relationship. This motivated us to add early stellar feedback.

As a check to see whether the addition of more energy or the timing of that energy addition had a stronger effect, we ran a series of simulations without the $10^{51}$ erg per supernova energy constraint. The best result from that series is represented by the cyan line and star. It shows that a $20 \%$ increase of the supernova energy lowered the stellar mass to the Moster et al. (2012) relationship. Its evolution more closely follows the $z=0$ Moster et al. (2012) relationship than the simulation using $10^{51} \mathrm{erg}$. Again, we refer the reader to 3.3 to see that the evolution does not follow what is described in Moster et al. (2012).

The yellow star and line show the reduced effect of stellar feedback when thermal diffusion is active for particles with their cooling shutoff. This requires the early stellar feedback efficiency, $\epsilon_{e s f}$, to be increased to 0.175 to produce a galaxy that matches the stellar mass-halo mass relation-

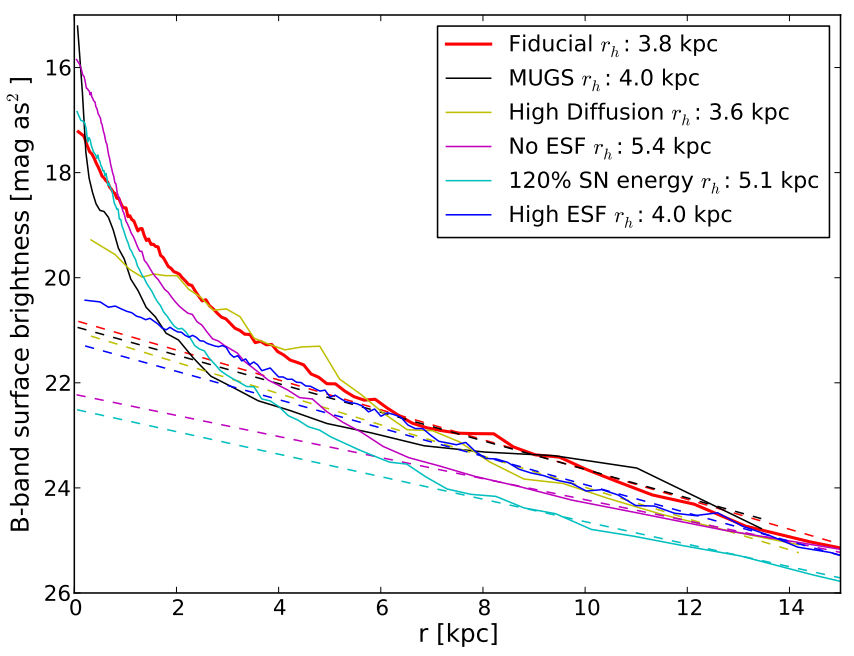

Figure 4. B-band surface brightness profile for g1536 simulated at a variety of feedback strengths. Each surface brightness profile has an exponential component with a central surface brightness around $21 \mathrm{mag} \operatorname{arcsec}^{-2}$. All the simulations that contain too many stars in Figure 3 have an exceptionally steep central profile that ends at a central surface brightness brighter than most observed galaxies. The galaxy that uses the MaGICC feedback deviates slightly from the pure exponential line, but does not increase nearly as quickly as the simulations with lower feedback.

ship at $z=0$. However, its evolution is much different from the other galaxies. Its stellar mass is increasing sharply at $z=0$. Figure 1 shows that its star formation rate is also dramatically increasing, so it will quickly overshoot the relationship. The drastic increase in star formation rate is the result of thermal feedback diffusing away instead of contributing to launching winds.

Simply reducing the thermal diffusion for all gas particles from 0.05 to 0.01 , as shown by the green star, required a corresponding reduction to $\epsilon_{\text {esf }}$ to form enough stars to match the stellar mass-halo mass relationship. However, the reduced thermal diffusion allowed cold clouds to condense out of the hot halo at the end of the simulation, which again led to a increasing star formation rate, though not as dramatic as in the higher thermal diffusion case.

As we made our parameter study, we found a degeneracy between $c^{\star}$ and $\epsilon_{e s f}$, so that a simulation where $c^{\star}=0.05$ and $\epsilon_{e s f}=0.15$ produces the same results as a simulation where $c^{\star}=0.1$ and $\epsilon_{e s f}=0.1$. Such a degeneracy is not surprising as the early feedback is immediate and limits star formation efficiency.

\subsection{Mass Distribution}

Having used the stellar mass-halo mass relationship as our constraint, we now examine other properties of the galaxies. Figure 4 shows surface brightness profiles produced using different amounts of stellar feedback in g1536. The one dimensional, face-on surface brightness profiles were constructed using the stellar population models from Girardi et al. (2010). Only stars in a disk with a radius of $15 \mathrm{kpc}$ and height $2 \mathrm{kpc}$ above and below the disk midplane are included. The profiles represent the mean values of 100 linearly spaced azimuthal bins. An exponential component 


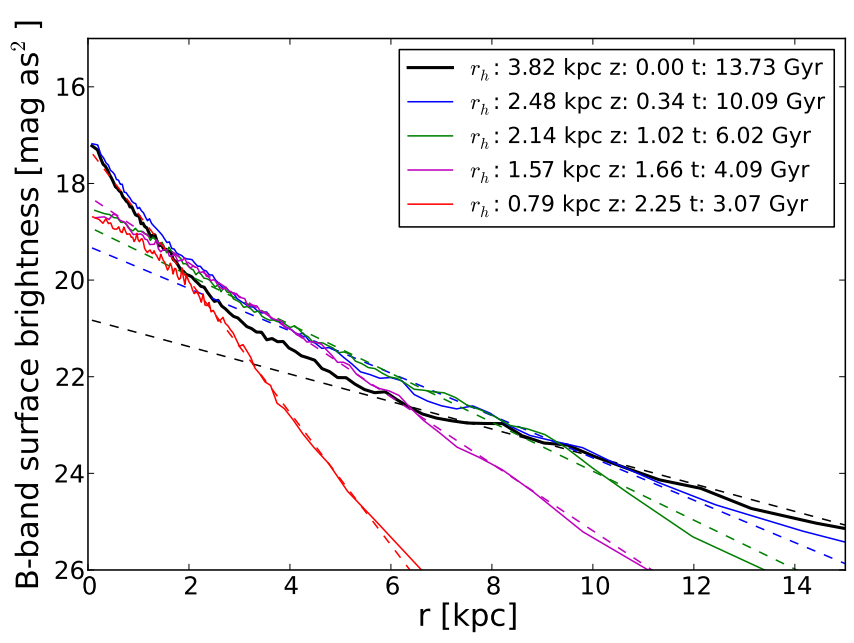

Figure 5. The evolution of the B-band surface brightness profile in the fiducial simulation.

of the disk was fit using stars with radii greater than $5 \mathrm{kpc}$. These fits are represented by the dashed lines the same color as the surface brightness profiles. Each exponential fit has a scale length around $4 \mathrm{kpc}$ and central surface brightnesses around 21 in the B-band, slightly brighter than the Freeman (1970) Law.

The fiducial simulation forms the right amount of stars and has an exponential surface brightness profile that steepens at $5 \mathrm{kpc}$ to reach a central $B$ surface brightness of $17 \mathrm{mag} \operatorname{arcsec}^{-2}$. By contrast, the simulations of g1536 without early stellar feedback each have exponential surface brightness profiles with slightly longer scale lengths but more extensive bulges. These bulges are characterized by higher Sersic index components in their inner regions that reach brighter central surface brightnesses than the simulations with early stellar feedback. This high central surface brightness is a common symptom of excess stellar mass in the central galactic region found in many galaxy simulations (Scannapieco et al. 2010; Stinson et al. 2010; Scannapieco et al. 2012).

Figure 5 shows the evolution of the $B$ surface brightness profile in our fiducial model. It shows the disk growing from the inside out. For the bulk of its evolution, the surface brightness profile remains exponential. In the final two time steps shown, an excess develops in the center. The scale length of the disk undergoes an evolution from $1 \mathrm{kpc}$ at $z=2$ to $4 \mathrm{kpc}$ at $z=0$.

To examine the underlying mass distribution of the galaxy, Figure 6 shows the rotation curves, the circular velocity, $v_{c}=\sqrt{G M(r) / r}$, as a function of radius, for the same galaxy models. The fiducial model has a nearly flat rotation curve. The rotation curve for the MUGS g1536 has a large central peak. The simulation without early stellar feedback also exhibits a high central peak in its rotation curve. However, the simulation with $20 \%$ more supernova energy has a much flatter rotation curve, though not as flat as the fiducial model.

The simulations with too much feedback have slowly rising rotation curves that are in conflict with the nearly flat rotation curve observed for the Milky Way and other

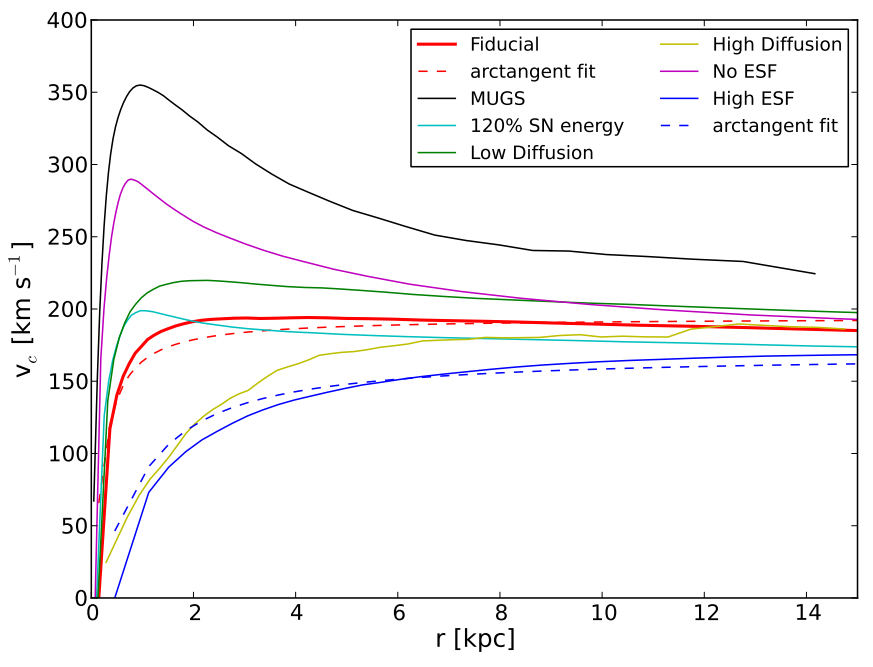

Figure 6. Plot of $v_{c}=\sqrt{G M(r) / r}$ as a function of $r$ for the all the models of g1536 and g5664 at $z=0$ modeled with varying amounts of stellar feedback.

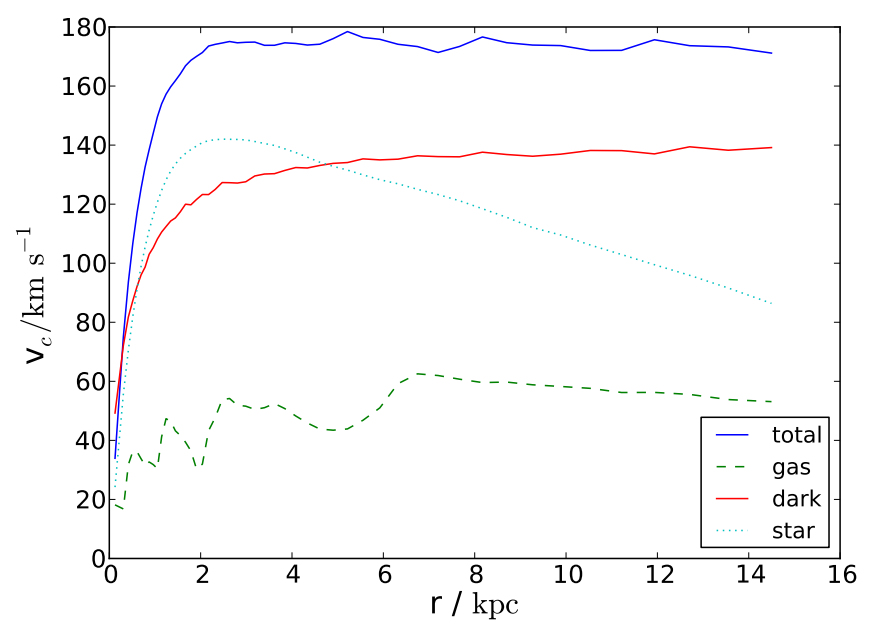

Figure 7. Plot of $v_{c}=\sqrt{G M(r) / r}$ as a function of $r$ for the simulated galaxy at $z=0$. Each component, dark matter (solid red), gas (green, dotted), and stars (light blue, dashed), is plotted separately to show the matter distributions of each component.

L* galaxies (Courteau 1997; de Blok et al. 2008). We have drawn sample model fits for the fiducial and high early stellar feedback simulations following Reves et al. (2011). They use $R_{T O}$ values, radius at which the profile turns over, of 0.25 and $1 \mathrm{kpc}$, respectively. The slowly rising rotation curves show the best agreement with the arctangent models used in Reyes et al. (2011), where typical $R_{T O}$ values are approximately the same as the disk scale length for galaxies with stellar masses similar to g1536's $2 \times 10^{10} \mathrm{M}_{\odot}$, which means that the rotation curves do not reach their peak until well beyond the disk scale length.

Even though the rotation curves slowly rise in the plotted region, the peak of the rotation curve, $180 \mathrm{~km} \mathrm{~s}^{-1}$ is still higher than the value of $v_{v i r}, 110 \mathrm{~km} \mathrm{~s}^{-1}$. In other words, the rotation curves turn over and decline outside the plotted region. 


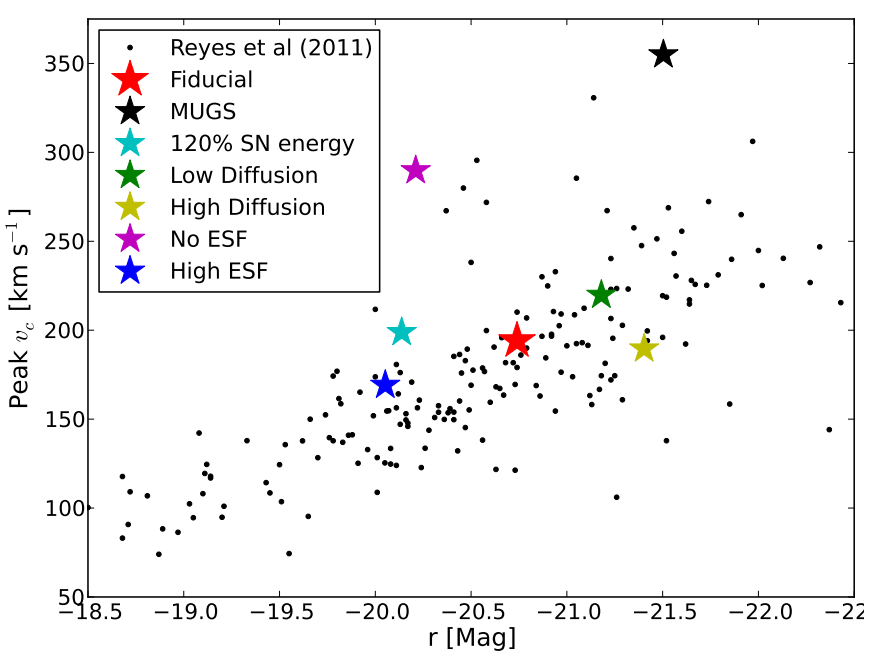

Figure 8. Peak circular velocity as a function of $r$-band magnitude compared to the Reves et al. (2011) observations. The simulations are plotted with the standard colour scheme used throughout this paper. The fiducial simulation (red) lies directly in the center of the observations. The simulations without early stellar feedback lie significantly above the observations.

The rotation curve for the fiducial galaxy is separated into the contributions from its constituent particle types in Figure 7 Stars dominate the potential in the central regions of the galaxy. The curves represent the mass included from just that component, so it does not represent the speed at which those particles are orbiting. Each component will orbit close to the total velocity.

The rotation velocity information enables another comparison between the amount of stars that have formed and the mass of the galaxy, the Tully-Fisher relationship (Tully \& Fisher 1977). The Tully-Fisher relationship compares the luminosity of a galaxy with its circular velocity. It is possible to argue for the choice of many different radii at which to measure the circular velocity. In Stinson et al. (2010), the galaxies were reported as fitting the Tully-Fisher relationship because the circular velocities were taken at 2.2 $R_{d}$, where $R_{d}$ is the scale radius, which was outside the central velocity peak. Here, we instead choose to compare our simulations with Reves et al. (2011), who use an arctangent model and report the peak velocities found using that fit. We compare these with the peak velocity from our simulations.

The fiducial simulation along with the other simulations that use early stellar feedback lie in the middle of the observed relationship. One exception is the high diffusion simulation, which has a bright $r$-band magnitude due to its significant late star formation but still has a low peak velocity. The simulations without early stellar feedback lie above most of the galaxies in the observed relationship due to their high central velocity peaks. No ESF and MUGS lie well above the observations, but $120 \%$ SN energy lies only slightly above the observations.

\subsection{Star Formation History}

The star formation history in Figure 9 shows that varying feedback has a significant impact on when stars form.

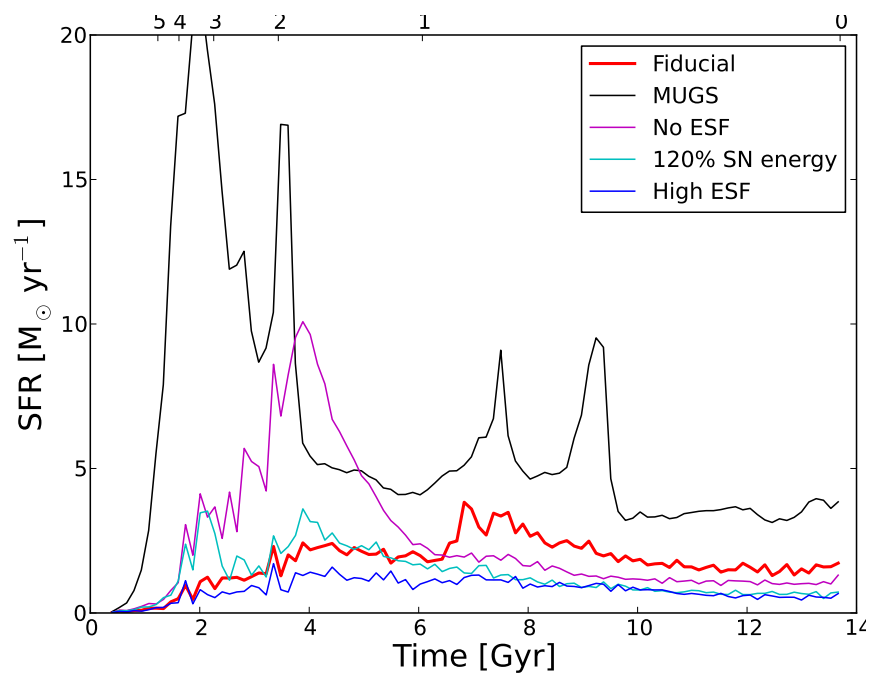

Figure 9. The star formation history of galaxies simulated with varying amounts of stellar feedback. Here, the simulation starts at Time $=0$ Gyr, on the left of this plot. The star formation rate is drastically reduced with the increased stellar feedback. The shape also changes from a quick rise and exponential falloff to a flatter evolution.

The MUGS case shows a fairly standard star formation history for most simulations reported in the literature (Scannapieco et al. 2012). Star formation follows the buildup of total halo mass as shown in Figure 11 All of the simulations without early stellar feedback experience a fairly broad peak of star formation just after 2 Gyr. This is the time period when many nearly equal mass mergers are rapidly building the halo mass. When the supernova energy is increased to $120 \%$, star formation after this peak is kept below $3 \mathrm{M}_{\odot} \mathrm{yr}^{-1}$. Introducing the early stellar feedback reduces or eliminates this burst from the simulations shown here because the early stellar feedback prevents gas from collapsing into the central $2 \mathrm{kpc}$ as shown in 4 Following the early peaks of star formation the star formation rate exponentially declines with intermittent bursts during mergers.

The star formation rate has been calculated using 100 bins each 137 Myr wide in Figure9. Figure1 shows the star formation rate calculated using 25 Myr bins, which shows the bursty nature of star formation that is characteristic of all of the galaxies outside of the MUGS. There are minor episodic bursts every 200-300 Myr overlaid onto the underlying shape of the star formation histories. These minor bursts correspond to the dynamical time of the galaxy, approximately the time it takes for gas to collapse after it is heated by stellar feedback (this phenomenon is described in more detail for dwarf galaxies in Stinson et al. 2007).

While the ESF galaxy does not show an increase in star formation at $z=4(2 \mathrm{Gyr})$, all the galaxies show a peak of star formation at $z=2(3.75 \mathrm{Gyr})$. This corresponds to the time of the first merger of halos with masses $>10^{11} \mathrm{M}_{\odot}$. The "no ESF" simulation (magenta line) with $E_{S N}=10^{51}$ erg but without early stellar feedback undergoes a moderately long, 2 Gyr starburst due to this merger. The fiducial simulation with early stellar feedback (red) has a shorter starburst $(\sim 100 \mathrm{Myr})$ and smaller amplitude $\left(8 \mathrm{M}_{\odot} \mathrm{yr}^{-1}\right)$ at this time. We note that a large population of small emis- 


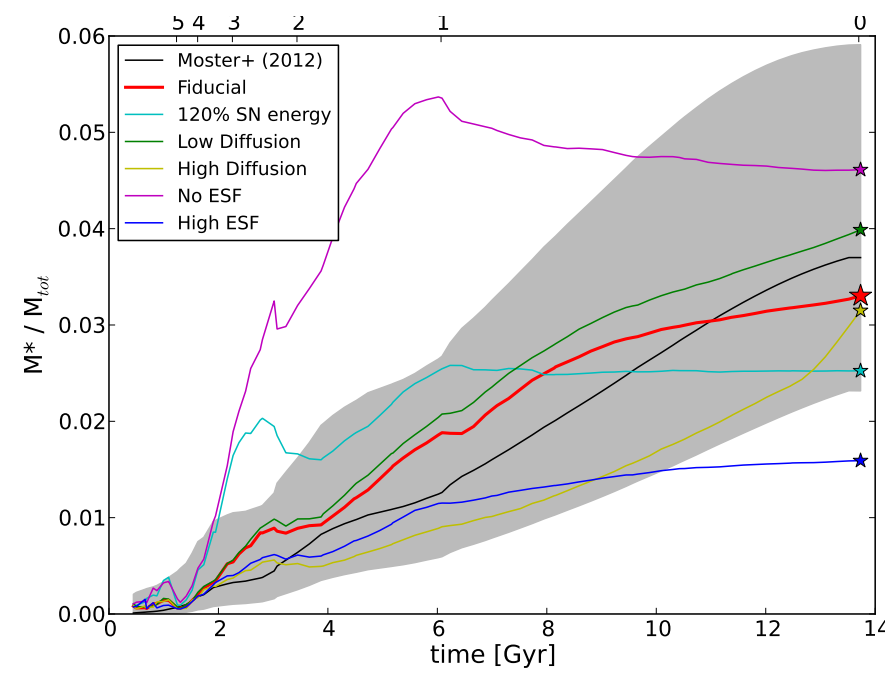

Figure 10. The evolution of the ratio of stellar mass to total halo mass for simulations using varying amounts of stellar feedback. Here, the simulation starts at Time $=0 \mathrm{Gyr}$, on the left of this plot. The black line is from Moster et al. (2012) that includes a detailed treatment of substructure to make the relationship between halo and stellar mass. The grey region represents the $1 \sigma$ variation in the model. The simulations evolve mostly within the $1 \sigma$ variation.

sion line galaxies has been detected at $z=2$ that have a major starburst as their first sign of significant star formation activity (van der Wel et al. 2011).

Shortly after the major merger, the fiducial simulation has another peak at $4 \mathrm{Gyr}$ that corresponds to the accretion of gas remaining from the $z=2$ merger. Its peak is lower and it lasts for a longer time. There are a couple of small, gas rich mergers that create a second star formation rate peak at 7 Gyr. This ends the merging history for g1536 and for the most part, the star formation rate slowly declines as star formation uses up the gas in the disk. The simulations without early stellar feedback decline more rapidly than the fiducial simulation and have a lower star formation rate for the last 7 Gyr of the simulation. This is due to those galaxies either using up their gas reservoirs faster (No ESF) or ejecting the gas further so that it is no longer available for star formation (120\% SN energy).

The star formation history of the fiducial simulation compares well to the star formation history of the Milky Way, though such studies have necessarily focused on the solar neighborhood (Rocha-Pinto \& Maciel 1997; Rocha-Pinto et al. 2000; Hernandez et al. 2000; Cignoni et al. 2006). These studies find that the solar neighborhood has a mostly flat star formation rate with factors of a few variation and a peak at $z \sim 1$. Looking at nearby dwarf galaxies, Weisz et al. (2011) finds that star formation histories are also mostly flat with variations limited to factors of a few, which again compares well with the simulation. We note that the galaxy in the Weisz et al. (2011) sample are all at least 3 magnitudes fainter than the simulated galaxies and have star formation rates two orders of magnitude lower. So, in a general sense, the star formation history of the fiducial simulation agrees well with the observations.

Figure 10 presents another view of the evolution of stellar mass. It shows the evolution of the ratio of stellar mass to total halo mass for simulations using varying amounts of stellar feedback. The black line shows the evolution of the ratio based on the evolution of the total halo mass inside $r_{200}$ according the recently published stellar mass-halo mass relationship in Moster et al. (2012). Moster et al. (2012) adds a detailed treatment of substructure to match the abundances between dark matter halo and stellar mass as well as using updated high redshift luminosity functions. The black line is calculated using the mass evolution of the g1536 halo including baryons. The grey region represents the $1 \sigma$ variation for the model. The simulations that use early stellar feedback evolve mostly within the $1 \sigma$ variation. The fiducial (red) simulation follows the black line most closely throughout the evolution of the galaxy. The simulations without early stellar feedback evolve outside the $1 \sigma$ variation early in their evolution because of the large amount of early star formation. A quick glance at Figure 9 indicates that the MUGS simulation that follows a star formation history typical of many published simulations would range even further outside the acceptable range of stellar masses at early times. The $120 \%$ SN energy simulation does not evolve very far outside the variance region, but it also does not follow the evolution of the mean line closely at all. It is well above the mean for the first $7 \mathrm{Gyr}$ and well below the mean for the last 5 Gyr. It is possible that this unique evolution has to do with the uniquely quiet merger history of g1536, but it could also indicate a mismatch. The high ESF model also evolves outside of the observed range of galaxy stellar masses. It forms fewer stars at $z=0$ than Moster et al. (2012) predict.

\subsection{Mass Evolution}

It is also possible to track the mass history for the other components of the galaxy. Figure 11] shows the mass history for four of the simulations. For each, the blue line represents the total galaxy mass inside $r_{v i r}$, where $r_{v i r}$ is the radius at which $\overline{\rho_{\text {gal }}}=390 \rho_{\text {background }}$. The black line is at one-sixth of the total mass, representing the cosmic fraction of baryons. The magenta line shows the actual mass of baryons inside the galaxy. The dashed cyan line represents the mass of gas with $\mathrm{T}<10^{5} \mathrm{~K}$ while the dash-dotted red line represents the mass for $\mathrm{T}>10^{5} \mathrm{~K}$, and the dotted green line shows the evolution of stellar mass. The total mass line clearly shows the two significant mergers at $z=2$ and $z=1$.

The origin of hot gas mass is coincident with the onset of star formation, indicating that stellar feedback could play a role in creating the hot halo; it may not only be accretion shocks as described in Toft et al. (2002) and Crain et al. (2010). Proving this claim is beyond the scope of this paper, so we leave it for future work. Both stellar mass and hot gas mass increase significantly during the $z=2$ merger, though the star formation increase is greatest in the case without early stellar feedback. The no ESF simulation also evolves to an equipartition between the three baryonic phases, stars, hot gas, and cold gas. It appears that the other simulations are converging to this equipartition, but it is hard to predict whether the stars will reach the same value as hot and cold gas.

In the two upper panels of simulations without thermal diffusion in particles with their cooling shut off, $25 \%$ of the baryons escape the potential well of the halo during the $z=2$ merger. Some baryons also initially escape in the 


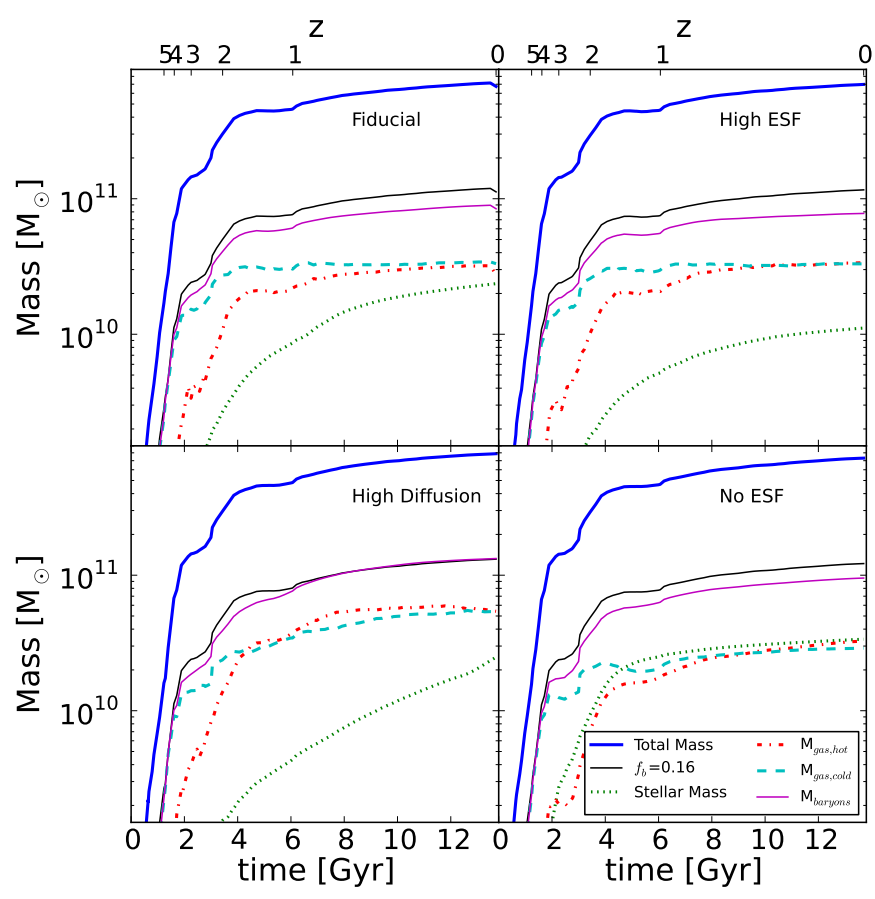

Figure 11. The mass evolution of the total halo mass (blue), stellar mass (dotted-green), cold gas $\left(\mathrm{T}<10^{5} \mathrm{~K}\right.$, dashed cyan), and hot $\left(\mathrm{T}>10^{5} \mathrm{~K}\right.$, dash-dotted red) gas within $r_{v i r}$ for the 4 simulations. The thin black line shows the cosmic baryon fraction of the total halo mass, while the thin magenta line shows the actual baryonic mass content of the halo.

simulations with thermal diffusion between all particles, but then baryons reaccrete.

\section{DISCUSSION}

The question remains about how the early stellar feedback more successfully reproduces observed galaxy properties than models without early stellar feedback. The greatest difference seems to be in the galaxy centers; the simulations with early stellar feedback have flat rotation curves while those without have central peaks. To examine this, Figure 12 compares the evolution of the inner $2 \mathrm{kpc}$ of the fiducial simulation with the $120 \%$ SN energy simulation. In Figure 9, it was shown that the $120 \%$ SN energy simulation forms more stars early throughout the galaxy than the fiducial simulation. Figure 12 shows that this pattern is replicated in the central $2 \mathrm{kpc}$, where most of the star formation is occurring. Figure 12 also shows that the higher star formation follows the higher mass of cold gas in the central region of the $120 \%$ SN energy simulation. So, there is a clear difference in the cold gas and star formation inside the inner 2 $\mathrm{kpc}$ of the two simulations.

What is causing the difference in cold gas in the center? There are several possiblities:

- Cold gas is more efficiently ejected from the early stellar feedback simulation

- Cold gas is replaced by hot gas in the inner $2 \mathrm{kpc}$.

- The increased thermal pressure supports the gas at distances from the center of the galaxy further than $2 \mathrm{kpc}$.

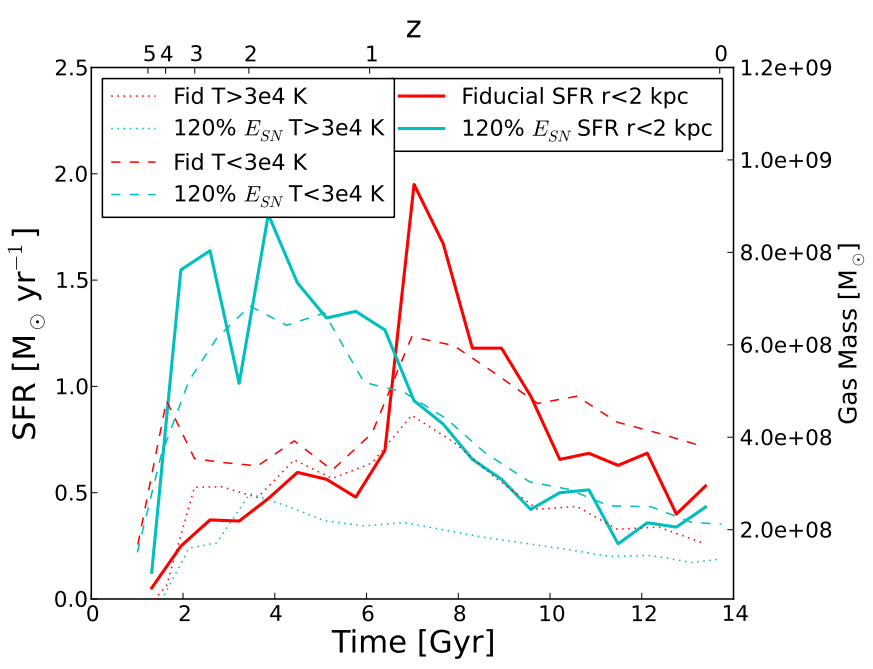

Figure 12. The evolution of the inner $2 \mathrm{kpc}$ of the stars and gas in the simulation for the fiducial (red) and $120 \%$ SN Energy (cyan) models. The solid lines show the star formation rate inside $2 \mathrm{kpc}$ throughout the simulation. The star formation rate scale is on the left y-axis. The dashed lines show the cold $\left(\mathrm{T}<3 \times 10^{4} \mathrm{~K}\right)$ gas mass evolution within $2 \mathrm{kpc}$. The dotted lines show the hot $\left(\mathrm{T}>3 \times 10^{4} \mathrm{~K}\right)$ gas mass evolution within $2 \mathrm{kpc}$. The gas masses are given on the right $\mathrm{y}$-axis.

Rather than the ESF simulation more efficiently ejecting gas, a look at the evolution of the galaxies shows that the $120 \%$ SN Energy ejects gas at higher velocity and to larger radii than the early stellar feedback simulation. The stronger gas ejection from the $120 \%$ SN Energy simulation is also apparent in the star formation history. The simulations without early stellar feedback have less star formation after $z \sim 1$ than those with early stellar feedback because they have less gas remaining in the central region.

Figure 12 also shows the mass of hot gas $\left(T>3 \times 10^{4}\right.$ $\mathrm{K}$, dotted lines) in the central $2 \mathrm{kpc}$ as a function of time. The hot gas mass is quite similar for the two simulations, so it is not $T>3 \times 10^{4}$ gas alone that is supporting gas from collapsing into the center.

Thus, we are left with the possibility that $T<3 \times 10^{4}$ $\mathrm{K}$ gas provides enough pressure to support gas to distances greater than $2 \mathrm{kpc}$. To check this possibility, Figure 13 shows the cumulative mass profile of gas at $z=2.25$ in the fiducial simulation compared with MUGS and the simulation that uses $120 \%$ SN Energy. $z=2.25$ is a time when there is a large discrepancy between the mass contained inside $2 \mathrm{kpc}$ for the simulations with and without early stellar feedback. There are also no satellites within $50 \mathrm{kpc}$ of the disk at $z=2.25$. Figure 13 shows that there is less gas inside the central 2 $\mathrm{kpc}$ of the fiducial simulation than the $120 \%$ SN Energy or MUGS simulations. This rapidly changes outside $3 \mathrm{kpc}$ as the early stellar feedback simulation has twice as much gas there. The increase extends from $3 \mathrm{kpc}$ well beyond $r_{v i r}$. In the case of the $120 \%$ SN Energy simulation, the missing gas has been ejected out that far as the cumulative mass catches up to the fiducial simulation at $300 \mathrm{kpc}$. In MUGS, ${ }^{b}$ http://www.mpia-hd.mpg.de/ stinson/magicc/movies/c.1td.05rp.1/close.mp4
versus http://www.mpia-hd.mpg.de/ stinson/magicc/movies/esn1.2/close.mp4 


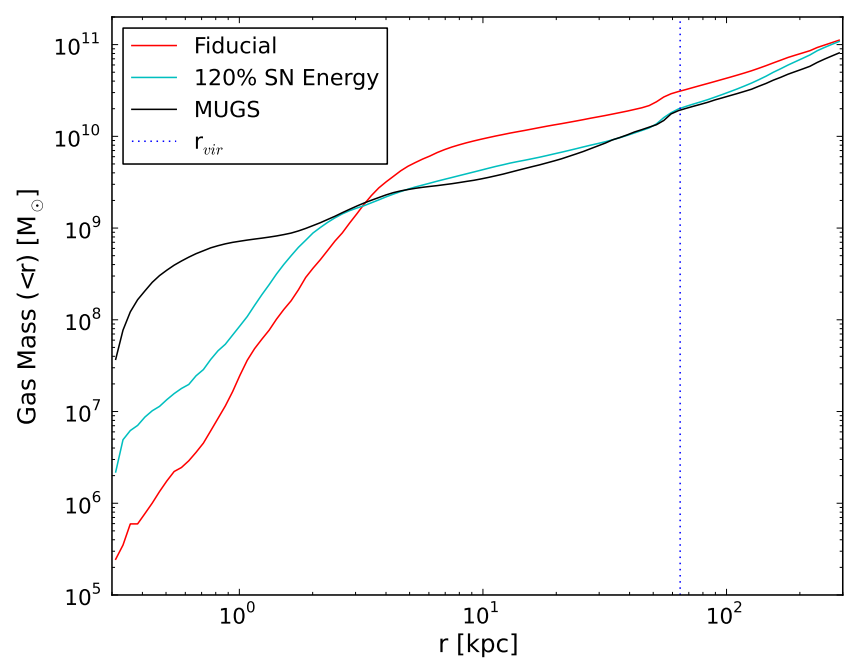

Figure 13. The cumulative gas mass profile at $z=2.25$ for the fiducial simulation compared to the $120 \%$ SN energy. The $120 \%$ SN energy simulation has up to ten times more mass at radii inside $3 \mathrm{kpc}$. In the fiducial simulation, this mass is supported outside $3 \mathrm{kpc}$.

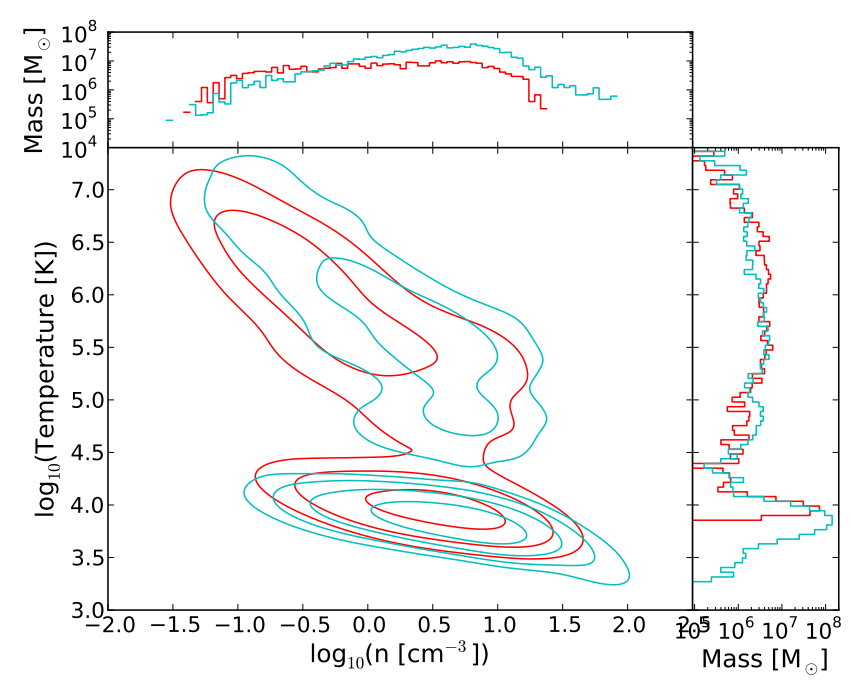

Figure 14. A comparison of the distribution of mass in temperature density phase space within $2 \mathrm{kpc}$ of the galaxy centre. The cyan contours represent the $120 \%$ SN Energy simulation distribution. The red contours show the fiducial simulation. There are ten contour levels placed at logarithmic mass intervals. Both the temperature axes have been collapsed into one-dimensional histograms along each axis.

however, the cumulative mass well below because the gas has been turned into stars.

To better understand why the fiducial simulation contains so much less gas inside $2 \mathrm{kpc}$ than the $120 \%$ SN Energy simulations, Figure 14 shows the temperature and density distributions for gas within $2 \mathrm{kpc}$ in the two simulations. Both the density and temperature distributions are projected into one-dimensional mass histograms in the top and right panels. The temperature mass histogram shows that the peak of the gas mass distribution is at ISM conditions, $T<3 \times 10^{4} \mathrm{~K}$, in both simulations. It also shows that the fiducial simulation has little gas at $T<10^{4} \mathrm{~K}$, whereas the $120 \%$ SN Energy simulation has a significant amount. This discrepancy shows how the early stellar feedback is having an effect similar to UV radiation by maintaining gas at $T>10^{4} \mathrm{~K}$. The UV heated gas at $10^{4} \mathrm{~K}$ will provide pressure support whereas the colder gas in the 120\% SN Energy simulation provides little. Consequently, the $120 \%$ SN Energy simulation contains more high density gas inside $2 \mathrm{kpc}$ including gas up to $100 \mathrm{~cm}^{-3}$.

The simulations have a similar amount of gas at $T>$ $3 \times 10^{4} \mathrm{~K}$, but the hot gas is lower density in the fiducial simulation. This shows that the thermal feedback gas is released in lower density regions in the fiducial simulation and has an easier time adiabatically expanding.

Our analysis shows the necessity for multiple forms of stellar feedback. Adding SN Energy alone simply blows stronger winds, but does not create sufficient $10^{4} \mathrm{~K}$ gas to provide pressure support in the disk that keeps low angular momentum gas out of the center of the disk. Without this feedback, the bulk of star formation happens too early. Thus, it seems that winds alone are insufficient to prevent the formation of galaxies with high central concentrations. It is also necessary for stellar feedback to create a warm component of the ISM. That said, the fiducial simulation exhibits the same angular momentum distribution described in Brook et al. (2012a), so outflows still play a key feedback role in shaping the galaxy.

\section{CONCLUSIONS}

We present a parameter study that varies the strength of stellar feedback in simulations of galaxy formation. As a constraint, we use the stellar mass-halo mass relationship. To reduce star formation enough to match the relationship, early stellar feedback from massive stars was required before they explode as supernova.

Our fiducial model best fits the stellar mass-halo mass relationship, the evolution of that relationship, has a flat rotation curve and an exponential surface brightness profile with a modest bulge in the center. In contrast to many previous cosmological galaxy formation simulations, most of the star formation occurs after $z=2$. There are two peaks of star formation that correspond to the two significant mergers to the galaxy. Both of these mergers are gas rich.

We find that the mass of stars formed at $z=0$ is very sensitive to the amount and timing of stellar feedback employed. Models that form too many stars follow a common pattern where they turn half of their baryons into stars, the other half is hot gas. These galaxies also form many of their stars at higher redshift than what Moster et al. (2012) find when they compare high redshift luminosity functions with N-body simulations. Galaxies that form too many stars have bright central concentrations of stars. This is reflected in galaxy rotation curves as a high central peak. Galaxies that form the right amount of stars have exponential surface brightness profiles and slowly rising rotation curves.

A simulation which does not use early stellar feedback, but increases the supernova energy to $1.2 \times 10^{51}$ erg ends up with a similar stellar mass to the fiducial simulation, but does not compare as well with other observed galaxy properties. The difference is that our implementation of early 
stellar feedback keeps the gas above $10^{4} \mathrm{~K}$ and at densities below $10 \mathrm{~cm}^{-3}$, an effect similar to that achieved by UV ionization. The low density, warm gas keeps the disk extended prior to $z=1$ and thus keeps gas out of the central $2 \mathrm{kpc}$ of the galaxy. Since less gas makes it to the central region, fewer stars form before $z=1$ and, therefore, this model does not result in the massive central concentration that forms without early stellar feedback.

The early stellar feedback produces galaxies that correspond to observations in a number of ways. Using early stellar feedback in dwarf galaxies spanning a wide mass range, Brook et al. (2012b) showed that the simulated galaxies match the disk scale length, gas fractions, and luminosities of observed galaxies. Brook et al. (2012a) has shown that the reason for such agreement is that outflows redistribute low angular momentum gas from the centers of galaxies to their outskirts. Stinson et al. (2012) showed that the outflows that redistribute the gas also populate the circumgalactic medium with an amount of oxygen that closely matches observations of OVI in the CGM. Macciò et al. (2012) showed that these outflows can also change the inner regions of dark matter density profiles from steeply rising cusps into flat cores. In Kannan et al (in prep), we will show how the stellar feedback described here performs in a larger sample of lower resolution galaxies in a cosmological volume. Early stellar feedback helps keeps gas out of the center of galaxies, which leads to forming disk galaxies like those that we observe.

\section{ACKNOWLEDGEMENTS}

We greatly appreciate the many suggestions the anonymous referee made to make this paper much better. The analysis was performed using the pynbody package (http://code.google.com/p/pynbody), which was written by Andrew Pontzen and Rok Roškar in addition to the authors. We thank Arjen van der Wel for useful conversations.

We are grateful to Ben Moster for providing his as yet unpublished stellar mass-halo mass relationship to us. The simulations were performed on the THEO cluster of the Max-Planck-Institut für Astronomie at the Rechenzentrum in Garching; the clusters hosted on SHARCNET, part of ComputeCanada. We greatly appreciate the contributions of these computing allocations. CBB acknowledges MaxPlanck-Institut für Astronomie for its hospitality and financial support through the Sonderforschungsbereich SFB 881 "The Milky Way System" (subproject A1) of the German Research Foundation (DFG). AVM and GS also acknowledge support from SFB 881 (subproject A1) of the DFG. JW and HMPC thank NSERC for support. TQ acknowledges support from grant AST-0908499 from the NSF.

\section{REFERENCES}

Agertz O., Moore B., Stadel J., Potter D., Miniati F., Read J., Mayer L., Gawryszczak A., Kravtsov A., Nordlund A., Pearce F., Quilis V., Rudd D., Springel V., Stone J., Tasker E., Teyssier R., Wadsley J., Walder R., 2007, MNRAS, 380, 963

Agertz O., Teyssier R., Moore B., 2011, MNRAS, 410, 1391
Balogh M. L., Pearce F. R., Bower R. G., Kay S. T., 2001, MNRAS, 326, 1228

Behroozi P. S., Conroy C., Wechsler R. H., 2010, ApJ, 717, 379

Benson A. J., Bower R. G., Frenk C. S., Lacey C. G., Baugh C. M., Cole S., 2003, ApJ, 599, 38

Birnboim Y., Dekel A., 2003, MNRAS, 345, 349

Bower R. G., Benson A. J., Crain R. A., 2012, MNRAS, 422, 2816

Bower R. G., Benson A. J., Malbon R., Helly J. C., Frenk C. S., Baugh C. M., Cole S., Lacey C. G., 2006, MNRAS, 370, 645

Bower R. G., McCarthy I. G., Benson A. J., 2008, MNRAS, 390, 1399

Brook C. B., Governato F., Roškar R., Stinson G., Brooks A. M., Wadsley J., Quinn T., Gibson B. K., Snaith O., Pilkington K., House E., Pontzen A., 2011, MNRAS, pp 595-+

Brook C. B., Kawata D., Gibson B. K., Freeman K. C., 2004, ApJ, 612, 894

Brook C. B., Stinson G., Gibson B. K., Roškar R., Wadsley J., Quinn T., 2012a, MNRAS, 419, 771

Brook C. B., Stinson G., Gibson B. K., Wadsley J., Quinn T., 2012b, MNRAS, 424, 1275

Chabrier G., 2003, PASP, 115, 763

Cignoni M., Degl'Innocenti S., Prada Moroni P. G., Shore S. N., 2006, A\&A, 459, 783

Conroy C., Wechsler R. H., 2009, ApJ, 696, 620

Conroy C., Wechsler R. H., Kravtsov A. V., 2006, ApJ, 647, 201

Courteau S., 1997, AJ, 114, 2402

Crain R. A., McCarthy I. G., Frenk C. S., Theuns T., Schaye J., 2010, MNRAS, 407, 1403

Dalla Vecchia C., Schaye J., 2008, MNRAS, 387, 1431

Dalla Vecchia C., Schaye J., 2012, ArXiv e-prints

de Blok W. J. G., Walter F., Brinks E., Trachternach C.,

Oh S.-H., Kennicutt Jr. R. C., 2008, AJ, 136, 2648

Dekel A., Silk J., 1986, ApJ, 303, 39

Durier F., Dalla Vecchia C., 2012, MNRAS, 419, 465

Dutton A. A., van den Bosch F. C., 2009, MNRAS, 396, 141

Ferland G. J., Korista K. T., Verner D. A., Ferguson J. W., Kingdon J. B., Verner E. M., 1998, PASP, 110, 761

Field G. B., 1965, ApJ, 142, 531

Field G. B., Goldsmith D. W., Habing H. J., 1969, ApJ, 155, L149

Freeman K. C., 1970, ApJ, 160, 811

Freyer T., Hensler G., Yorke H. W., 2006, ApJ, 638, 262

Gerritsen J. P. E., Icke V., 1997, A\&A, 325, 972

Girardi L., Williams B. F., Gilbert K. M., Rosenfield P., Dalcanton J. J., Marigo P., Boyer M. L., Dolphin A., Weisz D. R., Melbourne J., Olsen K. A. G., Seth A. C., Skillman E., 2010, ApJ, 724, 1030

Governato F., Brook C., Mayer L., Brooks A., Rhee G., Wadsley J., Jonsson P., Willman B., Stinson G., Quinn T., Madau P., 2010, Nature, 463, 203

Governato F., Mayer L., Wadsley J., Gardner J. P., Willman B., Hayashi E., Quinn T., Stadel J., Lake G., 2004, ApJ, 607, 688

Guedes J., Callegari S., Madau P., Mayer L., 2011, ApJ, 742,76 
Guo Q., White S., Li C., Boylan-Kolchin M., 2010, MNRAS, 404, 1111

Haardt F., Madau P., 2005, unpublished

Hernandez X., Valls-Gabaud D., Gilmore G., 2000, MNRAS, 316, 605

Hopkins P. F., Quataert E., Murray N., 2011, MNRAS, 417, 950

Jonsson P., 2006, MNRAS, 372, 2

Katz N., 1992, ApJ, 391, 502

Kaufmann T., Bullock J. S., Maller A. H., Fang T., Wadsley J., 2009, MNRAS, 396, 191

Kawata D., Gibson B. K., 2003, MNRAS, 340, 908

Kay S. T., Pearce F. R., Frenk C. S., Jenkins A., 2002, MNRAS, 330, 113

Klypin A., Prada F., 2009, ApJ, 690, 1488

Kroupa P., Tout C. A., Gilmore G., 1993, MNRAS, 262, 545

Leitherer C., Schaerer D., Goldader J. D., González Delgado R. M., Robert C., Kune D. F., de Mello D. F., Devost D., Heckman T. M., 1999, ApJS, 123, 3

Lupton R., Blanton M. R., Fekete G., Hogg D. W., O'Mullane W., Szalay A., Wherry N., 2004, PASP, 116, 133

Macciò A. V., Stinson G., Brook C. B., Wadsley J., Couchman H. M. P., Shen S., Gibson B. K., Quinn T., 2012, ApJ, 744, L9

Maller A. H., Bullock J. S., 2004, MNRAS, 355, 694

Mandelbaum R., Li C., Kauffmann G., White S. D. M., 2009, MNRAS, 393, 377

McCarthy I. G., Schaye J., Font A. S., Theuns T., Frenk C. S., Crain R. A., Dalla Vecchia C., 2012, ArXiv e-prints McKee C. F., Ostriker J. P., 1977, ApJ, 218, 148

More S., van den Bosch F. C., Cacciato M., Mo H. J., Yang X., Li R., 2009, MNRAS, 392, 801

More S., van den Bosch F. C., Cacciato M., Skibba R., Mo H. J., Yang X., 2011, MNRAS, 410, 210

Moster B. P., Naab T., White S. D. M., 2012, ArXiv eprints

Moster B. P., Somerville R. S., Maulbetsch C., van den Bosch F. C., Macciò A. V., Naab T., Oser L., 2010, ApJ, 710, 903

Murray N., Quataert E., Thompson T. A., 2010, ApJ, 709, 191

Navarro J. F., Benz W., 1991, ApJ, 380, 320

Oppenheimer B. D., Davé R., 2006, MNRAS, 373, 1265

Raiteri C. M., Villata M., Navarro J. F., 1996, A\&A, 315, 105

Rees M. J., Ostriker J. P., 1977, MNRAS, 179, 541

Reyes R., Mandelbaum R., Gunn J. E., Pizagno J., Lackner C. N., 2011, MNRAS, 417, 2347

Robertson B. E., Kravtsov A. V., 2008, ApJ, 680, 1083

Rocha-Pinto H. J., Maciel W. J., 1997, MNRAS, 289, 882

Rocha-Pinto H. J., Scalo J., Maciel W. J., Flynn C., 2000, A\&A, 358, 869

Sawala T., Guo Q., Scannapieco C., Jenkins A., White S., 2011, MNRAS, 413, 659

Scannapieco C., Gadotti D. A., Jonsson P., White S. D. M., 2010, MNRAS, 407, L41

Scannapieco C., Tissera P. B., White S. D. M., Springel V., 2008, MNRAS, 389, 1137

Scannapieco C., Wadepuhl M., Parry O. H., Navarro J. F., Jenkins A., Springel V., Teyssier R., Carlson E., Couch- man H. M. P., Crain R. A., Vecchia C. D., Frenk C. S., 2012, MNRAS, 423, 1726

Schaye J., Dalla Vecchia C., Booth C. M., Wiersma R. P. C., Theuns T., Haas M. R., Bertone S., Duffy A. R., McCarthy I. G., van de Voort F., 2010, MNRAS, 402, 1536

Schulz A. E., Mandelbaum R., Padmanabhan N., 2010, MNRAS, 408, 1463

Shen S., Wadsley J., Stinson G., 2010, MNRAS, 407, 1581

Slyz A. D., Devriendt J. E. G., Bryan G., Silk J., 2005, MNRAS, 356, 737

Somerville R. S., Primack J. R., 1999, MNRAS, 310, 1087

Springel V., Hernquist L., 2003, MNRAS, 339, 289

Stinson G., Seth A., Katz N., Wadsley J., Governato F., Quinn T., 2006, MNRAS, 373, 1074

Stinson G. S., Bailin J., Couchman H., Wadsley J., Shen S., Nickerson S., Brook C., Quinn T., 2010, MNRAS, 408, 812

Stinson G. S., Brook C., Prochaska J. X., Hennawi J., Shen S., Wadsley J., Pontzen A., Couchman H. M. P., Quinn T., Macciò A. V., Gibson B. K., 2012, MNRAS, 425, 1270 Stinson G. S., Dalcanton J. J., Quinn T., Kaufmann T., Wadsley J., 2007, ApJ, 667, 170

Sutherland R. S., Dopita M. A., 1993, ApJS, 88, 253

Thacker R. J., Couchman H. M. P., 2000, ApJ, 545, 728

Toft S., Rasmussen J., Sommer-Larsen J., Pedersen K., 2002, MNRAS, 335, 799

Torres G., Andersen J., Giménez A., 2010, A\&AR, 18, 67 Tully R. B., Fisher J. R., 1977, A\&A, 54, 661

van den Bosch F. C., Abel T., Croft R. A. C., Hernquist L., White S. D. M., 2002, ApJ, 576, 21

van der Wel A., Straughn A. N., Rix H.-W., Finkelstein S. L., Koekemoer A. M., Weiner B. J., Wuyts S., 2011, ApJ, 742, 111

Wadsley J. W., Stadel J., Quinn T., 2004, New Astronomy, 9,137

Wadsley J. W., Veeravalli G., Couchman H. M. P., 2008, MNRAS, 387, 427

Weisz D. R., Dalcanton J. J., Williams B. F., Gilbert K. M., Skillman E. D., Seth A. C., Dolphin A. E., McQuinn K. B. W., Gogarten S. M., Holtzman J., Rosema K., Cole A., Karachentsev I. D., Zaritsky D., 2011, ApJ, 739, 5

White S. D. M., Rees M. J., 1978, MNRAS, 183, 341 